\title{
Flood prediction in southern strip of Caspian Sea watershed.
}

\begin{abstract}
Modeling of hydrological process has become increasingly complicated since we need to take into consideration an increasing number of descriptive variables. Soil, topography, land-use, rainfall and flow are some of the variables which are difficult to be spatially measured. In recent years black box solutions like artificial neural networks have been used in modeling complex process of hydrologic events. The potential applications of multilayer feedforward back propagation neural networks for developing rainfall-runoff relationships for some homogeneous catchments located in the north of Iran were studied and compared with those of a multiple regression model. A total of 24 sites yielding 356 pairs of observed data were studied. The most popular network in hydrology, i.e., multilayer feedforward back propagation was used. Results show that among the different backpropagation learning algorithms used in this research, the Levenberg-Marquardt resulted in the best performance. Keywords: artificial neural networks, estimation of flood, flood frequency, hydrological modeling.
\end{abstract}

Keyword: Artificial neural networks; Estimation of flood; Flood frequency; Hydrological modeling. 